\title{
Diffusion-weighted magnetic resonance imaging for prediction of tumor response to neoadjuvant chemoradiotherapy using irinotecan plus $\mathbf{S}-1$ for rectal cancer
}

\author{
HIROSHI DOI ${ }^{1}$, NAOHITO BEPPU ${ }^{2}$, TAKASHI KATO ${ }^{1}$, MASASHI NODA ${ }^{2}$, \\ HIDENORI YANAGI $^{3}$, NAOHIRO TOMITA ${ }^{2}$, NORIHIKO KAMIKONYA ${ }^{1}$ and SHOZO HIROTA $^{1}$ \\ Departments of ${ }^{1}$ Radiology and ${ }^{2}$ Surgery, Hyogo College of Medicine, Nishinomiya, Hyogo 663-8501; \\ ${ }^{3}$ Department of Surgery, Meiwa Hospital, Nishinomiya, Hyogo 663-8186, Japan
}

Received April 20, 2015; Accepted June 22, 2015

DOI: $10.3892 / \mathrm{mco} .2015 .604$

\begin{abstract}
The purpose of this study was to investigate the clinical value of diffusion-weighted (DW) magnetic resonance imaging (MRI) as a predictor of tumor response in patients receiving neoadjuvant chemoradiotherapy (NA-CRT) for rectal cancer $(\mathrm{RC})$ through measurement of the apparent diffusion coefficient (ADC) value in each tumor. Neoadjuvant radiotherapy with a total dose of 45 Gy in 25 fractions was performed in all 16 patients with $\mathrm{RC}$, combined with irinotecan and S-1. MRI was performed before and after NA-CRT. Multiple factors were assessed to predict the pathological response to NA-CRT. The pathological response rate was determined in 9 patients $(56.3 \%)$. Statistical analyses indicated that the ADC value prior to NA-CRT was significantly lower in patients with a better response to NA-CRT $(\mathrm{P}=0.023)$. A cut-off value of $0.750 \times 10^{-3} \mathrm{~mm}^{2} / \mathrm{sec}$ obtained by a receiver operating characteristic curve analysis indicated a sensitivity of $77.8 \%$ and specificity of $85.7 \%$ for pathological responders to NA-CRT. In addition, the patients with lower ADC values exhibited a greater pathological response to NA-CRT $(\mathrm{P}=0.041)$. In conclusion, the ADC value of MRI of RC patients treated with NA-CRT followed by surgery may provide valuable information to predict the response to NA-CRT.
\end{abstract}

\section{Introduction}

Neoadjuvant chemoradiotherapy (NA-CRT) consisting of 45-50.4 Gy in 25-28 fractions followed by total mesorectal excision has been adopted as the standard treatment for

Correspondence to: Dr Hiroshi Doi, Department of Radiology, Hyogo College of Medicine, 1-1 Mukogawa-cho, Nishinomiya, Hyogo 663-8501, Japan

E-mail: h-doi@hyo-med.ac.jp

Key words: magnetic resonance imaging, apparent diffusion coefficient, diffusion-weighted magnetic resonance imaging, chemoradiotherapy, radiotherapy, S-1, rectal cancer, irinotecan, preoperative radiotherapy, neoadjuvant chemoradiotherapy locally advanced rectal cancer (RC) (1-6). The addition of chemotherapy to neoadjuvant radiotherapy (NA-RT) has been demonstrated to be feasible, with enhanced antitumor effects (7). Moreover, the use of 5-fluorouracil (5-FU)-based chemotherapy has gained widespread acceptance for the treatment of locally advanced rectal adenocarcinoma $(1,2)$.

S-1 is a novel oral anticancer drug composed of tegafur, 5-chloro-2,4-dihydroxypyridine, oteracil (which was designed to enhance the oral efficacy of tegafur) and a prodrug of 5-FU. Several clinical studies have demonstrated that NA-CRT combined with $\mathrm{S}-1$ is associated with mild toxicity and exhibits an efficacy equivalent to that of other CRT regimens used for RC (8-12). Clinical studies of irinotecan (CPT-11) plus S-1 combination therapy have been reported to produce non-inferiority outcomes for metastatic colorectal cancer. In addition, several clinical studies have demonstrated that NA-RT combined with S-1 is associated with mild toxicity and an efficacy equivalent to that achieved by other systemic chemotherapies (13).

Diffusion-weighted (DW) magnetic resonance imaging (MRI) is a non-invasive functional MRI technique that is sensitive to the mobility of water protons in biological tissues, which is dependent on a number of factors, such as cell density, vascularity, the viscosity of the extracellular fluid and cell membrane integrity (14-16). The apparent diffusion coefficient (ADC) calculated from DW-MRI measurements may be used to quantify and express these properties. The majority of the studies have demonstrated that the pretreatment ADC is negatively correlated with response to treatment (17-28). It appears that necrotic areas with high pretreatment ADC values may be less sensitive to CRT. However, several studies have produced contradictory results $(29,30)$. Previously published data on the value of DW-MRI as a predictive tool for anticancer treatment responses in patients with $\mathrm{RC}$ are scarce and conflicting. In addition, there are no reports on the correlation between MRI and the pathological response to NA-CRT using CPT-11 and S1.

Therefore, this study was conducted to investigate the clinical value of DW-MRI as a predictor of tumor response in patients receiving NA-CRT for $\mathrm{RC}$ through the measurement of the tumor ADC. 


\section{Patients and methods}

Patients. Patients who were treated with NA-RT (45 Gy in 25 fractions) followed by surgery for primary $\mathrm{RC}$ and who underwent MRI, including DW-MRI, before and after NA-CRT prior to surgery at the of Hyogo College of Medicine Hospital between July, 2011 and March, 2014 were included in this study. The study endpoints included the predictive factors for the pathological response to NA-CRT for RC using MRI. The patient eligibility criteria were as follows: Age $\geq 20$ years, histologically confirmed primary adenocarcinoma of the rectum and no evidence of metastatic disease in distant organs. A patient with cT2N1M0 RC and a patient with cT3N2bM1a disease were included in this analysis based on experienced physicians' decisions. A total of 16 patients were analyzed in the present study. The patient characteristics are summarized in Table I.

Preoperative clinical staging included clinical assessment, computed tomography (CT) scans between the chest and whole pelvis, a pelvic MRI, a full blood analysis and colonoscopy with biopsy. The study protocol was approved by the Ethics Committee of Hyogo College of Medicine and all the patients provided written informed consent prior to NA-CRT.

Treatment protocol. The protocol of NA-CRT applied for the present study with minor modifications was recently described in detail (13). Briefly, all the patients were placed in the supine position and helically scanned on an Aquilion LB CT unit (Toshiba, Otawara-shi, Japan). For each patient, a planning CT scan of the entire pelvis from the lower abdomen to below the ischial tuberosities was obtained at 5-mm intervals. The CT dataset was transferred to the Focus $\mathrm{XiO}^{\mathrm{TM}}$ treatment-planning system (CMS, Inc., St Louis, MO, USA) to outline the volumes of interest.

The gross target volume (GTV) included the primary rectal tumor and nodal metastases. The clinical target volume (CTV) comprised the GTV with a 1-cm margin, as well as the perirectal, obturator and internal iliac nodes. The planning target volume (PTV) was the CTV with a $0.5-\mathrm{cm}$ margin. Furthermore, there was an additional 7-mm leaf margin to the PTV, in order to cover the PTV more homogeneously.

RT was performed using a 3D conformal RT technique, which was typically performed with a 4-field box technique using $10 \mathrm{MV}$ photons. The planned RT was delivered using an Elekta Synergy device (Elekta, Crawley, UK). The patients were treated with a dose of 1.8 Gy daily up to a total dose of $45 \mathrm{~Gy}$ in 25 fractions. S-1 was administered orally at a dose of 120 (100-140) mg/body/day on days 1-5, 8-12, 22-26 and 29-33. CPT-11 was delivered at a dose of $60(60-80) \mathrm{mg} / \mathrm{m}^{2} /$ day on days 1, 8, 22 and 29. Surgery was performed 6-10 weeks after the completion of RT. The removed specimens were pathologically evaluated.

MRI technique and analysis. An MRI of the pelvis was routinely performed prior to treatment (pre-CRT) but not after NA-CRT (post-CRT). In the present study, all the patients had both pre- and post-CRT MRI data, including DW imaging. Rectal MRI was performed using Magnetom Avanto (Siemens Medical Solutions, Erlangen, Germany), Intera 1.5T (Philips Healthcare, Amsterdam, The Netherlands) and
Table I. Patient characteristics.

\begin{tabular}{|c|c|}
\hline Variables & Values \\
\hline Total no. of patients & 16 \\
\hline Age, years [median (range)] & $62.5(47-79)$ \\
\hline \multicolumn{2}{|l|}{ Gender, no. } \\
\hline Male & 13 \\
\hline Female & 3 \\
\hline \multicolumn{2}{|l|}{ Performance status, no. } \\
\hline 0 & 16 \\
\hline \multicolumn{2}{|l|}{ Histopathology, no. (\%) } \\
\hline Tub 1 & $8(50.0)$ \\
\hline Tub 2 & $8(50.0)$ \\
\hline \multicolumn{2}{|l|}{ Radiotherapy, no. (\%) } \\
\hline 45 Gy in 25 fractions (1.8 Gy/fr) & $16(100.0)$ \\
\hline Treatment term, days (range) & $37(29-51)$ \\
\hline \multicolumn{2}{|l|}{ Chemotherapy, no. (\%) } \\
\hline S-1 plus CPT-11 & $16(100.0)$ \\
\hline \multicolumn{2}{|l|}{ Terms, days (range) } \\
\hline Pre-MRI to start of RT & $21(3-48)$ \\
\hline Completion of RT to post-MRI & $31.5(10-45)$ \\
\hline Completion of RT to surgery & $42(54-69)$ \\
\hline Post-MRI to surgery & $24(11-42)$ \\
\hline \multicolumn{2}{|l|}{ Clinical stage, no. } \\
\hline $\mathrm{T} 2$ & 1 \\
\hline T3 & 12 \\
\hline $\mathrm{T} 4$ & 3 \\
\hline No & 6 \\
\hline N1 & 8 \\
\hline $\mathrm{N} 2$ & 2 \\
\hline M0 & 15 \\
\hline $\mathrm{M} \mathrm{a}^{\mathrm{a}}$ & 1 \\
\hline IIA & 6 \\
\hline IIC & 1 \\
\hline IIIA & 1 \\
\hline IIIB & 5 \\
\hline IIIC & 2 \\
\hline IVA & 1 \\
\hline
\end{tabular}

aPara-aortic lymph node metastasis. Tub 1, well-differentiated adenocarcinoma; Tub 2, moderately differentiated adenocarcinoma; MRI, magnetic resonance imaging; RT, radiotherapy; CPT-11, irinotecan.

Magnetom Skyra 3T (Siemens Medical Solutions, Erlangen, Germany) in 12, 19 and 1 scanning(s), respectively. The MR images were evaluated by a picture archiving and communication system and analyzed by a single radiologist with 5 years of clinical experience who was blinded to the other results and the study design. Without any information regarding the patient, the sum of the longest diameters (SLD) of rectal tumors for the tumor sizes and ADC values was calculated by manually tracing the tumor boundaries on axial post-gadolinium T1-weighted MR images or axial T2-weighted MR images 
and by placing oval-shaped regions of interest (ROI) on axial DW images. The ROIs were manually drawn within the tumor on axial images with a b value of $1,000 \mathrm{sec} / \mathrm{mm}^{2}$ on the selected ADC maps. Each ROI was designed to have $4.00 \mathrm{~mm}^{2}$ in the rectal tumor. Four ROIs were generated for each patient and the mean of the ADC values in the ROIs was considered to be the ADC value of the tumor.

The pre-CRT SLD (baseline SLD) and post-CRT SLD were compared according to the Response Evaluation Criteria in Solid Tumors guidelines (31). The \% decrease of each tumor was calculated as follows:

$$
\left[\left(\mathrm{SLD}_{\text {pre-CRT }}-\mathrm{SLD}_{\text {post-CRT }}\right) / \mathrm{SLD}_{\text {pre-CRT }}\right] \times 100
$$

In addition, a tumor reduction rate of $\geq 30 \%$ was considered to be a partial response. The $\triangle \mathrm{ADC}$ value, defined as the percentage of the difference from the pre-CRT to the post-CRT ADC was calculated using the formula:

$$
\Delta \mathrm{ADC}(\%)=\left[\left(\mathrm{ADC}_{\text {post-CRT }}-\mathrm{ADC}_{\text {pre-CRT }}\right) / \mathrm{ADC}_{\text {pre-CRT }}\right] \times 100
$$

Pathological examination of the surgical specimens. Pathological staging was conducted according to the tumor-node-metastasis staging system. The pathological tumor stage was compared with the clinical stage in each patient. Tumor downstaging was defined as a lower pathological stage compared with the clinical stage prior to treatment. The tumor response after CRT was determined according to the following pathological grading: Grade 0, not effective; grade 1a, high response in $<1 / 3$ cancer cells; grade $1 b$, high response in $1 / 3-2 / 3$ cancer cells; grade 2 , high response in $>2 / 3$ cancer cells; grade 3 , complete response. In addition, patients with grade 2 or 3 tumor response were defined as pathological responders and patients with grade $1 \mathrm{a}$ or $1 \mathrm{~b}$ tumor response as pathological non-responders (32).

Statistical analysis. The patient data were recorded on standardized forms, reviewed and expressed as median and range, unless otherwise indicated. The duration between events was calculated from the day of surgery to the day of confirmation of an event. Cumulative local control, disease-free survival and overall survival estimates were calculated using the Kaplan-Meier method. As regards the association between the two groups, continuous variables and incidence of patients, the trend for incidence was assessed using the Mann-Whitney $\mathrm{U}$ test, the Fisher's exact test and Chi-square test for trend, respectively. The optimal cut-off value was determined using receiver operating characteristic (ROC) curve analysis. All the analyses were performed using the GraphPad Prism 6.0b software program (GraphPad Software, Inc., San Diego, CA, USA). P-values of $<0.05$ were considered to indicate statistically significant differences.

\section{Results}

$A D C$ values pre- and post-CRT. The baseline SLD was $105.0 \mathrm{~mm}$ (range, 59.0-200.0 $\mathrm{mm}$ ). The percent decrease of SLD and the number of patients who exhibited PR on MRI was $33.2 \%$ (range, 1.7-67.0\%) and 9 (56.3\%), respectively. The results of the ADC measurement are shown in Table II. The ADC values were significantly increased following CRT $(\mathrm{P}<0.0001)$. In addition, there were no significant differences in the ROIs for the ADC values between pre- and
Table II. ADC values in MRI findings

\begin{tabular}{lc}
\hline ADC & Values (range) \\
\hline Pre-CRT & \\
Value $\left(\times 10^{-3} \mathrm{~mm}^{2} / \mathrm{sec}\right)$ & $0.753(0.613-0.869)$ \\
Area of ROI, $\mathrm{mm}^{2}$ & $4.03(3.77-4.18)$ \\
Post-CRT & \\
Value $\left(\mathrm{x} 10^{-3} \mathrm{~mm}^{2} / \mathrm{sec}\right)$ & $1.114(0.856-1.799)$ \\
Area of ROI, $\mathrm{mm}^{2}$ & $4.05(3.82-4.39)$ \\
$\Delta \mathrm{ADC}, \%$ & $55.8(3.4-174.2)$
\end{tabular}

ADC, apparent diffusion coefficient; MRI, magnetic resonance imaging; CRT, chemoradiotherapy; ROI, region of interest.

post-CRT ADCs $(\mathrm{P}=0.7732)$. When compared with the clinical stage, downstaging was achieved in $6(37.5 \%), 8$ (50.0\%) and 8 patients $(50.0 \%)$ in the $\mathrm{T}$ stage, $\mathrm{N}$ stage and stage grouping, respectively. As regards pathological findings, 4, 3, 8 and 1 patient(s) had grade 1a, 1b, 2 and 3 disease, respectively, and the pathological response rate was $56.3 \%$.

Predictive factors of the pathological response to NA-CRT. To assess the potential factors that may predict the pathological response to NA-CRT in RC, we analyzed various factors between the non-responder and the responder groups. The results of the analysis are presented in Table III. The ADC values following CRT were higher compared with those prior to CRT in all the patients. In addition, the pre-CRT ADC value was significantly lower in the responder group $(\mathrm{P}=0.023)$. The diagnostic performance of pre-CRT ADC in the prediction of the response to CRT was evaluated using the ROC curve analysis (Fig. 1). With a cut-off value of $0.750 \mathrm{~mm}^{2} / \mathrm{sec}$, we obtained a sensitivity and specificity for pathological responders of 77.8 and $85.7 \%$, respectively. In addition, the area under the ROC curve was $84.1 \%$. The patients with pre-CRT ADC values $\leq 0.750 \mathrm{~mm}^{2} / \mathrm{sec}$ included 7 responders $(87.5 \%)$; there were significantly more responders with pre-CRT ADC values $\leq 0.750 \mathrm{~mm}^{2} / \mathrm{sec}(\mathrm{P}=0.041$, Fig. 2).

\section{Discussion}

NA-CRT followed by surgery is currently the standard treatment for locally advanced RC. However, a proportion of patients benefit little from NA-CRT. Therefore, the prediction of the response to NA-CRT is crucial.

DW-MRI is a non-invasive functional MRI technique that has been shown to predict the response to NA-CRT in $\mathrm{RC}$. Lambregts et al (28) previously reported that the quantitative evaluation of the ADC may be used as a biomarker for the response to treatment. The diagnostic performance of the ADC with various cut-off values ranging between 1.2 and $1.4 \times 10^{3} \mathrm{~mm}^{2} / \mathrm{sec}$ in $\mathrm{RC}$ has been reported to be equivalent to $\sim 46-100 \%$ sensitivity and $\sim 56-84 \%$ specificity $(18,19,21,29)$. The value of DW-MRI as a predictive tool for assessing response to NA-CRT in patients with RC is currently poorly understood. In addition, there is yet no 
Table III. Predictive factors for the pathological response to NA-CRT.

\begin{tabular}{|c|c|c|c|}
\hline Factors & Non-responders & Responders & P-value \\
\hline No. of patients & 7 & 9 & \\
\hline Gender, no. & & & 0.550 \\
\hline Male & 5 & 8 & \\
\hline Female & 2 & 1 & \\
\hline Clinical T stage, no. & & & 0.897 \\
\hline 2 & 0 & 1 & \\
\hline 3 & 6 & 6 & \\
\hline 4 & 1 & 2 & \\
\hline T downstaging, no. (\%) & $2(28.6)$ & $4(44.4)$ & 0.633 \\
\hline Baseline sum diameter, mm (range) & $104(59-129)$ & $106(82-200)$ & 0.423 \\
\hline Sum diameter shrinkage, $\%$ (range) & $27.5(1.7-65.9)$ & $37.7(14.2-67.0)$ & 0.585 \\
\hline Pre-CRT ADC, $\times 10^{-3} \mathrm{~mm}^{2} / \mathrm{sec}$ (range) & $0.822(0.747-0.869)$ & $0.718(0.613-0.852)$ & 0.023 \\
\hline Post-CRT ADC, $\times 10^{-3} \mathrm{~mm}^{2} / \mathrm{sec}$ (range) & $1.153(0.921-1.799)$ & $1.031(0.856-1.680)$ & 0.598 \\
\hline$\triangle \mathrm{ADC}$ (range) & $0.391(0.120-1.070)$ & $0.557(0.034-1.742)$ & 0.656 \\
\hline
\end{tabular}

NA-CRT, neoadjuvant chemoradiotherapy; CRT, chemoradiotherapy; ADC, apparent diffusion coefficient.

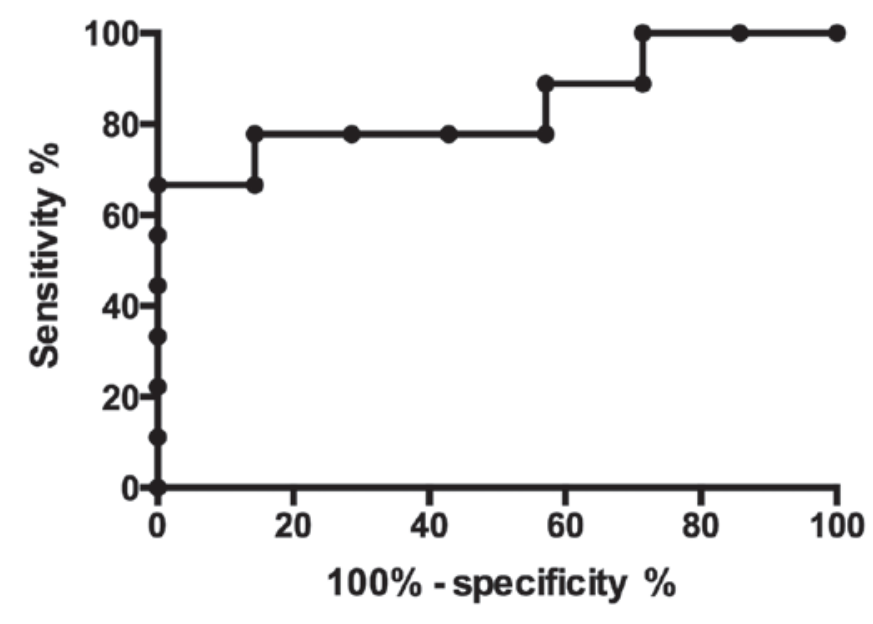

Figure 1. The receiver operating characteristic (ROC) curve analysis demonstrated that the area under the ROC curve was $84.1 \%(\mathrm{P}=0.023)$.

consensus on the true clinical value of ADC measurement for response assessment in $\mathrm{RC}$.

In this study, we analyzed the pre- and post-CRT ADC values and the pathological findings in surgically removed specimens in order to assess the response to treatment. Our findings demonstrated that the pre-CRT ADC value was strongly correlated with the response to CRT using S-1 and CPT-11. In addition, we obtained a cut-off value of $0.750 \times 10^{3} \mathrm{~mm}^{2} / \mathrm{sec}$ that exhibited high sensitivity and specificity. Moreover, the post-CRT ADC value and the changes in ADC values following CRT did not significantly affect the pathological response to NA-CRT. Further investigation with a large number of patients should be performed to assess the clinical availability of ADC values and other findings in MRI.

The increase in the ADC value and the tumor shrinkage following NA-CRT were not found to be correlated with the pathological response to CRT in the present study. Differences

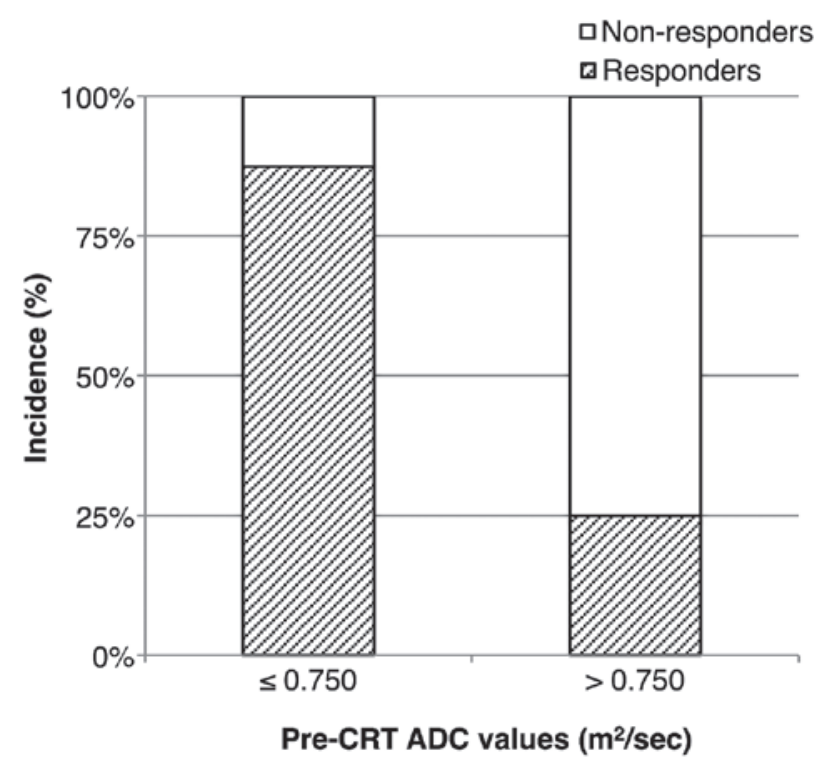

Figure 2. The 8 patients with ADC values $\leq 0.750\left(\times 10^{-3} \mathrm{~mm}^{2} / \mathrm{sec}\right)$ included 7 patients $(87.5 \%)$ who exhibited grade 2 or 3 response in the resected tumors. The remaining 8 patients with ADC values $>0.750\left(\times 10^{-3} \mathrm{~mm}^{2} / \mathrm{sec}\right)$ included 2 patients $(25.0 \%)$ who exhibited grade 2 or 3 response in the resected tumors Patients with ADC values $\leq 0.750\left(\times 10^{-3} \mathrm{~mm}^{2} / \mathrm{sec}\right)$ significantly responded to neoadjuvant chemoradiotherapy $(\mathrm{P}=0.041)$. CRT, chemoradiotherapy; ADC, apparent diffusion coefficient.

between previous reported results and our findings are likely associated with differences in the study design $(3,6,14-18)$.

There were several limitations to the present study. First, we analyzed a limited number of patients who received NA-CRT, including S-1 plus CPT-11 therapy, and the ROI size may exert a considerable effect on the tumor ADC values. Lambregts et al (28) reported that the small-sample ROI measurements, similar to our method, exhibited significantly smaller variance compared with the whole-volume ROIs, 
which were generated by freehand. In addition, sampling ROIs in the tumor may minimize susceptibility artifacts due to air-tissue interfaces in the rectum, and may simplify the application of quantitative DW imaging. Therefore, the sample ROI measurement appears to be straightforward for clinical use, while requiring less time compared to the whole-volume measurements. The timing of MRI varied before and after NA-CRT, which may lead to different results. However, we believe that the pre-CRT ADC value was not affected by the time variance. Moreover, a phase II clinical trial is currently ongoing to evaluate the efficacy and safety of NA-CRT using S-1 and CPT-11. The ADC analysis based on the present study will therefore be performed with a large number of patients and the role of MRI on the survival will be also investigated in the near future.

A relatively high $b$ value of $1,000 \mathrm{sec} / \mathrm{m}^{2}$ may eliminate possible microvascular contamination of the computed ADC values and may improve the detection of slow-moving water molecules or small diffusion distances, which are essential in the early response evaluations. We qualitatively analyzed the ADC values with a focus on the primary tumor, as it is difficult to match the histopathological analysis of the lymph nodes site by site with that of the MRI, mainly due to the depletion of mesorectal lymph nodes following irradiation on pathological analysis. We recently reported that the response of the primary tumor to NA-CRT was correlated with the number of positive nodes on pathological analysis in patients with RC who received NA-CRT followed by surgery (33). The prediction of the pathological response of involved lymph nodes to NA-CRT is poorly understood. The correlation between the ADC values and pathological response of metastatic nodes should be further investigated in order to assess the potential to minimize surgery with abbreviating lymph node dissection.

Another approach to NA-RT commonly in use for RC is short-course RT (25 Gy in 5 fractions) followed by surgery within 1 week $(2,3)$. We recently reported the feasibility and validity of a novel protocol using modified short-course RT combined with S-1 followed by surgery (10-12). Further studies are required to standardize a method of quantitative analysis of DW imaging in order to determine the definitive clinical application of this technique in patients with RC treated with short-course RT followed by surgery.

In conclusion, we demonstrated that the ADC value of MRI in RC may provide valuable information enabling the prediction of the response to CRT including CPT-11 plus S-1. This method appears to have the potential to facilitate the physicians' decision-making process when selecting patients to be treated with CRT or surgery alone.

\section{References}

1. National Comprehensive Cancer Network. Rectal Cancer, Version 3, 2015. http://www.nccn.org/professionals/physician gls/pdf/rectal.pdf. Accessed June 23, 2015.

2. Glimelius B, Tiret E, Cervantes A and Arnold D; ESMO Guidelines Working Group: Rectal cancer: ESMO Clinical Practice Guidelines for diagnosis, treatment and follow-up. Ann Oncol 24 (Suppl 6): vi81-vi88, 2013.

3. Marijnen CA and Glimelius B: The role of radiotherapy in rectal cancer. Eur J Cancer 38: 943-952, 2002.

4. Sauer R, Becker H, Hohenberger W, et al; German Rectal Cancer Study Group: Preoperative versus postoperative chemoradiotherapy for rectal cancer. N Engl J Med 351: 1731-1740, 2004.
5. Wagman R, Minsky BD, Cohen AM, Guillem JG and Paty PP: Sphincter preservation in rectal cancer with preoperative radiation therapy and coloanal anastomosis: Long term follow-up. Int J Radiat Oncol Biol Phys 42: 51-57, 1998.

6. Frykholm GJ, Isacsson U, Nygård K, et al: Preoperative radiotherapy in rectal carcinoma - aspects of acute adverse effects and radiation technique. Int J Radiat Oncol Biol Phys 35: 1039-1048, 1996.

7. Ceelen W, Fierens K, Van Nieuwenhove Y and Pattyn P: Preoperative chemoradiation versus radiation alone for stage II and III resectable rectal cancer: A systematic review and meta-analysis. Int J Cancer 124: 2966-2972, 2009.

8. Wada H, Nemoto K, Nomiya T, et al: A phase I trial of S-1 with concurrent radiotherapy in patients with locally recurrent rectal cancer. Int J Clin Oncol 18: 273-278, 2013.

9. Morimoto S, Shimada M, Kurita N, et al: Preoperative radiotherapy combined with S-1 for advanced lower rectal cancer: Phase I trial. Hepatogastroenterology 59: 1428-1432, 2012.

10. Doi H, Beppu N, Odawara S, et al: Neoadjuvant short-course hyperfractionated accelerated radiotherapy (SC-HART) combined with S-1 for locally advanced rectal cancer. J Radiat Res (Tokyo) 54: 1118-1124, 2013.

11. Beppu N, Matsubara N, Noda M, et al: The timing of surgery after preoperative short-course S-1 chemoradiotherapy with delayed surgery for T3 lower rectal cancer. Int J Colorectal Dis 29: 1459-1466, 2014.

12. Beppu N, Matsubara N, Kakuno A, et al: Feasibility of modified short-course radiotherapy combined with a chemoradiosensitizer for T3 rectal cancer. Dis Colon Rectum 58: 479-487, 2015.

13. Sato T, Ozawa H, Hatate K, et al: A Phase II trial of neoadjuvant preoperative chemoradiotherapy with $\mathrm{S}-1$ plus irinotecan and radiation in patients with locally advanced rectal cancer: Clinical feasibility and response rate. Int J Radiat Oncol Biol Phys 79: 677-683, 2011.

14. Chenevert TL, Stegman LD, Taylor JM, et al: Diffusion magnetic resonance imaging: An early surrogate marker of therapeutic efficacy in brain tumors. J Natl Cancer Inst 92: 2029-2036, 2000.

15. Hamstra DA, Rehemtulla A and Ross BD: Diffusion magnetic resonance imaging: A biomarker for treatment response in oncology. J Clin Oncol 25: 4104-4109, 2007.

16. DeSouza NM, Riches SF, Vanas NJ, et al: Diffusion-weighted magnetic resonance imaging: A potential non-invasive marker of tumour aggressiveness in localized prostate cancer. Clin Radiol 63: 774-782, 2008

17. Kim SH, Lee JM, Hong SH, et al: Locally advanced rectal cancer: Added value of diffusion-weighted MR imaging in the evaluation of tumor response to neoadjuvant chemo- and radiation therapy. Radiology 253: 116-125, 2009.

18. Monguzzi L, Ippolito D, Bernasconi DP, et al: Locally advanced rectal cancer: Value of ADC mapping in prediction of tumor response to radiochemotherapy. Eur J Radiol 82: 234-240, 2013.

19. Lambregts DM, Maas M, Riedl RG, et al: Value of ADC measurements for nodal staging after chemoradiation in locally advanced rectal cancer - a per lesion validation study. Eur Radiol 21: 265-273, 2011.

20. Curvo-Semedo L, Lambregts DM, Maas M, et al: Rectal cancer: Assessment of complete response to preoperative combined radiation therapy with chemotherapy - conventional MR volumetry versus diffusion-weighted MR imaging. Radiology 260: 734-743, 2011.

21. Cai G, Xu Y,Zhu J, Gu WL, Zhang S, Ma XJ, Cai SJ and Zhang Z: Diffusion-weighted magnetic resonance imaging for predicting the response of rectal cancer to neoadjuvant concurrent chemoradiation. World J Gastroenterol 19: 5520-5527, 2013.

22. Dzik-Jurasz A, Domenig C, George M, et al: Diffusion MRI for prediction of response of rectal cancer to chemoradiation. Lancet 360: 307-308, 2002.

23. Barbaro B, Vitale R, Valentini V, et al: Diffusion-weighted magnetic resonance imaging in monitoring rectal cancer response to neoadjuvant chemoradiotherapy. Int J Radiat Oncol Biol Phys 83: 594-599, 2012.

24. Ha HI, Kim AY, Yu CS, Park SH and Ha HK: Locally advanced rectal cancer: Diffusion-weighted MR tumour volumetry and the apparent diffusion coefficient for evaluating complete remission after preoperative chemoradiation therapy. Eur Radiol 23: 3345-3353, 2013.

25. Lambregts DM, Vandecaveye V, Barbaro B, et al: Diffusion-weighted MRI for selection of complete responders after chemoradiation for locally advanced rectal cancer: A multicenter study. Ann Surg Oncol 18: 2224-2231, 2011. 
26. Kim SH, Lee JY, Lee JM, Han JK and Choi BI: Apparent diffusion coefficient for evaluating tumour response to neoadjuvant chemoradiation therapy for locally advanced rectal cancer. Eur Radiol 21: 987-995, 2011.

27. Lambrecht M, Deroose C, Roels S, et al: The use of FDG-PET/CT and diffusion-weighted magnetic resonance imaging for response prediction before, during and after preoperative chemoradiotherapy for rectal cancer. Acta Oncol 49: 956-963, 2010.

28. Lambregts DM, Beets GL, Maas M, et al: Tumour ADC measurements in rectal cancer: Effect of ROI methods on ADC values and interobserver variability. Eur Radiol 21: 2567-2574, 2011.

29. Kim JC, Lim JS, Keum KC, et al: Comparison of diffusion-weighted MRI and MR volumetry in the evaluation of early treatment outcomes after preoperative chemoradiotherapy for locally advanced rectal cancer. J Magn Reson Imaging 34: 570-576, 2011.
30. DeVries AF, Kremser C, Hein PA, et al: Tumor microcirculation and diffusion predict therapy outcome for primary rectal carcinoma. Int J Radiat Oncol Biol Phys 56: 958-965, 2003.

31. Eisenhauer EA, Therasse P, Bogaerts J, et al: New response evaluation criteria in solid tumours: Revised RECIST guideline (v1.1). Eur J Cancer 45: 228-247, 2009.

32. Toiyama Y, Inoue Y, Saigusa S, et al: C-reactive protein as predictor of recurrence in patients with rectal cancer undergoing chemoradiotherapy followed by surgery. Anticancer Res 33: 5065-5074, 2013.

33. Beppu N, Matsubara N, Noda M, et al: Pathologic evaluation of the response of mesorectal positive nodes to preoperative chemoradiotherapy in patients with rectal cancer. Surgery 157: 743-751, 2015. 